\title{
0 efeito da interferência contextual em idosos
}

\author{
Wesley R. Gonçalves ${ }^{1,3}$ \\ Guilherme M. Lage 2,3 \\ Alexandro B. da Silva ${ }^{3}$ \\ Herbert Ugrinowitsch ${ }^{3}$ \\ Rodolfo N. Benda ${ }^{3}$
}

https://doi.org/10.5628/rpcd.07.02.217

\author{
${ }^{1}$ Universidade Presidente Antonio Carlos (UNIPAC) \\ Conselheiro Lafaiete, MG, Brasil \\ 2 Universidade FUMEC \\ Belo Horizonte, MG, Brasil \\ ${ }^{3}$ Gedam (Grupo de Estudos em Desenvolvimento e \\ Aprendizagem Motora) \\ Escola de Educação Física, \\ Fisioterapia e Terapia Ocupacional \\ Universidade Federal de Minas Gerais (UFMG) \\ Brasil
}

\section{ABSTRACT \\ The contextual interference effect in elderly people}

O presente estudo teve como objetivo verificar o efeito da interferência contextual (EIC) na aquisição de habilidades motoras em idosos. Foi utilizada uma tarefa de posicionamento manual, caracterizada pelo transporte de três bolas de tênis em uma seqüência e tempo alvo pré-determinados. $\mathrm{O}$ experimento constou de 4 fases: 1) aquisição, 2) transferência 1 (T1), 3) transferência 2 (T2) e 4) retenção da aquisição. Os sujeitos foram divididos aleatoriamente em quatro grupos $(n=12)$ : grupo de prática aleatória-aleatória (A-A), que realizou a tarefa de forma aleatória na aquisição e na retenção; grupo de prática aleatória-blo$\cos (\mathrm{A}-\mathrm{B})$, que realizou a aquisição de forma aleatória e a retenção em blocos; grupo de prática em blocos-blocos (B-B), que realizou a aquisição e a retenção em blocos; grupo de prática em blocos-aleatória (B-A), que realizou a aquisição em blocos e a retenção de forma aleatória. Os resultados mostraram que um dos grupos que praticou em regime aleatório apresentou-se mais variável durante a fase de aquisição que os grupos que praticaram em blocos. Entretanto, este mesmo grupo aleatório, no primeiro bloco do teste de retenção da aquisição, mostrou-se mais preciso que um dos grupos em blocos. Esses resultados confirmaram parcialmente o EIC em idosos.

Palavras-chave: aprendizagem motora, estrutura de prática, interferência contextual.
The purpose of this study was to investigate the contextual interference effect (CIE) in the acquisition of motor skills in elderly people. A manual positioning task was used, it was characterized by the transport of three tennis ball in a movement sequence and predetermined target times. The experiment consisted of 4 phases: 1) acquisition, 2) transfer 1 (T1), 3) transfer 2 (T2) and 4) retention of the acquisition. The subjects were randomly distributed into four groups: random-random group $(R-R)$, which performed the tasks in a random order in the acquisition and retention; random-blocked group $(R-B)$, which performed the acquisition in a random order and the retention in a blocked order; blocked-blocked group (B-B), which performed the tasks in a blocked order in the acquisition and retention; blocked-random group $(B-R)$, which performed the acquisition in a blocked order and the retention in a random order. The results showed that one of the random groups was more variable during the acquisition compared to the both blocked groups. However, this random group, in the first block of the retention was more precise than one of the blocked groups. These results partially confirm the CIE in elderly people.

Key-words: motor learning, structure of practice, contextual interference 


\section{INTRODUÇÃO}

Uma das variáveis de maior influência na aprendizagem motora é a estruturação da prática. A forma como a prática é organizada interfere na qualidade e na quantidade de informações recebidas, processadas e geradas através das restrições impostas ao aprendiz. A área de estudo que investiga os efeitos da ordem de execução de duas ou mais habilidades na aprendizagem motora é conhecida como interferência contextual.

A interferência contextual foi primeiramente definida por Battig(2), como a interferência produzida por outras tarefas e as formas pelas quais elas são processadas. De forma geral, os estudos sobre interferência contextual comparam os efeitos das práticas variadas em blocos e aleatória $(18,19,22,24)$. A prática em blocos, considerada de baixa interferência contextual, caracteriza-se pela execução de todas as tentativas de uma determinada habilidade para então iniciar-se as execuções da seguinte (ex. WWW XXX YYY). Já a prática aleatória, considerada de alta interferência contextual, é baseada em uma ordem de execução não sistemática das habilidades a serem praticadas (ex. X W Y W X Y X W Y) (5). Apesar de a prática em blocos produzir melhor performance durante a fase de aquisição, quando a performance dos grupos é comparada nos testes de retenção e transferência, a prática aleatória conduz a um melhor desempenho que a prática em blocos ${ }^{(14)}$. A maioria dos estudos que investigou o efeito da interferência contextual (EIC) contou com crianças, adolescentes ou adultos como sujeitos ${ }^{(1,22,24)}$. Dick, Beth, Shankle, Dick-Muehlke, Cotman e Kean (7) e Dick, Hsieh, Dick-Muehlke, Daves e Cotman ${ }^{(8)}$ investigaram a hipótese da variabilidade de prática entre idosos saudáveis e portadores do mal de Alzeimer. Entretanto, apenas o trabalho de Dick, Hsieh, Dick-Muehlke, Daves e Cotman ${ }^{(8)}$ seguiu os delineamentos dos estudos da área de aprendizagem motora, incluindo os testes de retenção e transferência e permitiu, devido ao delineamento utilizado e resultados encontrados, fazer algumas inferências quanto ao EIC em idosos. Participaram desse trabalho 56 idosos saudáveis e 56 idosos portadores do mal de Alzeimer distribuídos em grupos controle, de prática constante, prática em blocos e prática aleatória consistindo de fases de aquisição, transferência próxima, transferência distante ou intermediária e retenção, em uma tarefa de arremessar a um alvo a diferentes distâncias. Os resultados mostraram que os sujeitos portadores do mal de Alzeimer se beneficiaram apenas da prática constante e não conseguiram em nenhum outro regime de prática uma transferência para uma nova tarefa com um nível de dificuldade intermediário. Entre os sujeitos saudáveis, o grupo de prática aleatória apresentou melhor performance que o grupo em blocos na fase de retenção e foi o único grupo a apresentar desempenho superior ao grupo controle durante a fase de transferência distante. No trabalho de Dick, Hsieh, Dick-Muehlke, Daves e Cotman ${ }^{(8)}$ o grupo de prática aleatória não alternava a distância de lançamento a cada tentativa devido à dificuldade de entendimento apresentada pelo grupo portador do mal de Alzeimer. O grupo de prática aleatória limitou-se então, em alternar as distâncias de forma não hierárquica depois de um quarto das tentativas. Esse procedimento pode ter contribuído para um enfraquecimento do efeito da interferência contextual.

Dois estudos encontrados preocuparam-se especificamente em investigar o EIC em idosos(4, 6). Del Rey ${ }^{(6)}$ verificou a relação entre nível de atividade física e interferência contextual. O estudo contou com 48 mulheres idosas, distribuídas em grupos de praticantes e não praticantes de atividade física, que realizaram uma tarefa de timing coincidente em regime de prática aleatória e em blocos. Na fase de aquisição, os grupos que praticaram em blocos apresentaram-se mais precisos e menos variáveis. Na interação entre velocidade do estímulo, nível de atividade e contexto de aquisição, para análise do erro constante, verificou-se que nos sujeitos ativos a aquisição de forma aleatória facilitou a retenção da aquisição nas velocidades consideradas mais difíceis (as velocidades mais baixas) para aprendizagem da tarefa investigada (5 e $7 \mathrm{mph}$ ) Esses resultados deram suporte para o EIC entre idosos ativos e para relação entre nível de atividade física e função cognitiva em idosos. Nesse trabalho não foi aplicado teste de transferência, o que não permitiu verificar a capacidade de adaptação dessa população a uma nova tarefa, considerando o regime de prática.

Carnahan, Vandervoort e Swanson(4), com o objetivo de discutir o EIC em função dos efeitos da idade na 
aquisição de habilidades, contaram com um grupo de jovens além de um grupo de idosos com 24 sujeitos divididos em grupos de prática aleatória e em blocos. A tarefa do estudo foi pressionar, o mais rápido o possível, três diferentes seqüências pré-determinadas de um conjunto de quatro teclas na região alfanumérica de um computador. O teste de retenção da aquisição foi aplicado com metade das tentativas executadas de forma aleatória e metade de forma em blocos. O teste de transferência, como no estudo de Del Rey(6), também não foi aplicado por Carnahan e colegas(4). Os resultados apontaram para um limitado efeito da interferência contextual em idosos, pois apenas na fase de retenção e apenas em uma seqüência de movimentos, na seqüência teoricamente mais difícil segundo os autores do estudo, foi verificado o EIC, com o grupo de prática aleatória executando a tarefa mais rápido que o grupo de prática em blocos.

Com o avanço da idade, o desempenho motor e cognitivo decresce e a prática de atividade física pode interferir positivamente, minimizando as perdas decorrentes da terceira idade(20,21). Apesar de serem mais lentos nas medidas de desempenho, devido possivelmente a déficits de processamento central, como dificuldade na seleção das informações, dificuldades em ignorar informações irrelevantes e na tomada de decisão, os idosos apresentam capacidade de se adaptar tanto às demandas ambientais quanto à aprendizagem motora $(9,12)$. Todavia, pouco se sabe como a estruturação da prática pode contribuir para a aprendizagem nessa população.

Assim, o objetivo deste estudo é verificar o EIC na aprendizagem de uma tarefa de posicionamento manual em idosos.

\section{MATERIAL E MÉTODO}

A amostra foi constituída por 48 sujeitos de ambos os sexos, com idade média de $66,45 \pm 4,89$ anos, todos participantes de um programa de atividades físicas da Escola de Educação Física, Fisioterapia e Terapia Ocupacional (UFMG). Os sujeitos não relataram qualquer patologia e nunca haviam tido contato com a tarefa utilizada. Todos assinaram um termo de consentimento livre e esclarecido para participarem do estudo.

Foi utilizada uma tarefa de posicionamento manual, caracterizada pelo transporte de três bolas de tênis em uma seqüência e tempo alvo pré-determinados entre 3 conjuntos de 2 recipientes de uma plataforma identificados com as letras A, B e C. Uma central de controle ligada a um microcomputador, constituída por um conjunto de diodos (estímulo visual para o início da tarefa) e uma chave de respostas (controle dos tempos para início e fim da tarefa) foi controlada por um software que mediu os tempos de reação, de movimento e de resposta em cada tentativa e armazenou esses dados (Figura 1).

Antes de cada tentativa, era fornecida a informação da seqüência de movimentos a ser realizada através da apresentação de um cartão de $8 \times 11 \mathrm{~cm}$. Ao sinal "prepara", fornecido pelo experimentador, a chave era pressionada, e, após um estímulo visual (acendimento dos diodos), a chave era solta, medindo o tempo de reação, devendo então se iniciar o transporte das bolas de tênis com a mão dominante na seqüência pré-definida em determinado tempo alvo. Ao término da seqüência de posicionamento das bolas, a chave era pressionada novamente medindo o tempo de movimento e caracterizando o fim da tarefa.

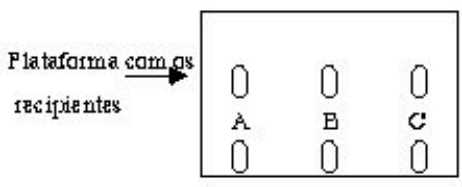

Chempatador

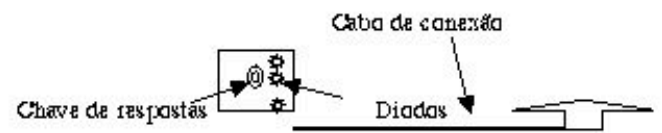

Figura 1. Esquema do aparelho utilizado no experimento.

Os sujeitos foram designados aleatoriamente para quatro grupos experimentais $(n=12)$ : grupo de prática aleatória-aleatória (A-A), que realizou a tarefa de forma aleatória na aquisição e na retenção da aquisição; grupo de prática aleatória-blocos (A-B), que realizou a aquisição de forma aleatória e a retenção da aquisição em blocos; grupo de prática em blocos-blocos (B-B), que realizou a aquisição e a retenção da aquisição em blocos; grupo de prática em blocos-aleatória (B-A), que realizou a aquisição em blocos e a retenção da aquisição de forma aleatória. 
O experimento constou de quatro fases: 1 ) aquisição, 2) transferência $1(\mathrm{~T} 1), 3)$ transferência 2 (T2) e 4) retenção da aquisição (R). Na aquisição, os grupos praticaram 45 tentativas de três diferentes seqüências de movimento (A/B/C; C/B/A; B/C/A) em um tempo alvo de $3.500 \mathrm{~ms}$, sendo que os grupos B-B e B-A praticaram em blocos, executando 15 tentativas consecutivas de cada tarefa, na ordem apresentada acima, e os grupos A-A e A-B praticaram aleatoriamente, não repetindo consecutivamente a mesma tarefa ao longo da fase de aquisição. Antes de iniciar a fase de prática, cada sujeito, sem restrição de tempo, realizou uma tentativa de cada seqüência de movimentos para familiarização com o equipamento e os procedimentos.

Foi fornecido feedback qualitativo em direção e magnitude para todos os sujeitos cinco segundos após o final de cada tentativa válida durante a fase de aquisição. Se o sujeito realizou a tarefa exatamente no tempo alvo foi dito: "você acertou", se o sujeito apresentou o erro absoluto até $100 \mathrm{~ms}$, foi dito: "você está próximo", se o erro absoluto foi entre 100 e $250 \mathrm{~ms}$ foi dito, conforme o caso: "você está um pouco atrasado" ou "você está um pouco adiantado" e caso o erro absoluto tenha sido superior a $250 \mathrm{~ms}$ do tempo alvo, foi dito, de acordo com a direção desse erro: "você está muito atrasado" ou "você está muito adiantado".

O teste de transferência (T1) foi realizado três minutos após a aquisição, no qual os sujeitos realizaram 12 tentativas de uma nova tarefa (C/A/B), com complex idade similar, em termos de números de componentes envolvidos na execução, às tarefas da aquisição e no mesmo tempo alvo, sem fornecimento de feedback. O segundo teste de transferência (T2) foi realizado três minutos após o término do primeiro, no qual os sujeitos realizaram 12 tentativas de uma nova seqüência $(C / A / B / A)$, sem fornecimento de feedback, com um tempo alvo de $4.500 \mathrm{~ms}$ e complexidade maior que as anteriores, pois possuía quatro componentes, consistindo no retorno da bola do recipiente A para sua posição original.

No teste de retenção da aquisição foram realizadas 12 tentativas das mesmas seqüências $(\mathrm{A} / \mathrm{B} / \mathrm{C}$; $\mathrm{C} / \mathrm{B} / \mathrm{A} ; \mathrm{B} / \mathrm{C} / \mathrm{A}$ ) praticadas na fase de aquisição, com o mesmo tempo alvo e sem o fornecimento de feed- back. Ressalta-se que, apesar das seqüências da fase de retenção da aquisição serem as mesmas da fase de aquisição, os grupos B-A e A-B realizaram a fase de retenção da aquisição com um regime de prática inverso ao que tinham praticado na aquisição (Tabela 1). Esse procedimento foi aplicado no sentido de analisar a influência da especificidade da prática durante o teste de retenção e a capacidade de adaptação de um regime de prática a outro(18).

Tabela 1. Regime de prática dos grupos experimentais nas fases de aquisição e retenção da aquisição.

\begin{tabular}{ccc}
\hline Grupos experimentais & $\begin{array}{c}\text { Ordem de execução das tarefas na fase } \\
\text { de aquisição e teste de retenção }\end{array}$ \\
\hline & Aquisição & Retenção \\
\hline A-A & Aleatória & Aleatória \\
A-B & Aleatória & Blocos \\
B-B & Blocos & Blocos \\
B-A & Blocos & Aleatória \\
\hline
\end{tabular}

As variáveis dependentes analisadas nesse estudo foram as médias do erro absoluto (ms) e as médias do desvio-padrão do erro absoluto (ms).

Os dados da fase de aquisição foram organizados em nove blocos de cinco tentativas cada. Os testes de transferência e retenção da aquisição foram organizados em blocos com média de seis tentativas cada. Isto se justifica pela escolha da execução de duas tentativas de cada uma da seqüências de movimento, tanto na estrutura de prática que foi aplicada na fase de aquisição, como na estrutura de prática não vivenciada na aquisição.

\section{RESULTADOS}

\section{Erro Absoluto}

Para a fase de aquisição (Figura 1), foi conduzida uma ANOVA two-way (4 grupos x 9 blocos) com medidas repetidas no segundo fator que não indicou diferença significante entre grupos $[\mathrm{F}(3,44)=0,44$, $\mathrm{p}=0,72]$, assim como não indicou interação entre grupos e blocos $[\mathrm{F}(24,352)=1,25, \mathrm{p}=0,19]$. Houve diferença significante entre blocos $[\mathrm{F}(8,24)=24,08$, $\mathrm{p}<0,001]$ e o teste Post Hoc de Tukey registrou diferença entre o primeiro e os demais blocos de tentativas $(p<0,001)$. 
Para comparação entre o último bloco de tentativas da fase de aquisição e os testes foi conduzida uma ANOVA two-way (4 grupos x 7 blocos) com medidas repetidas no segundo fator que não indicou diferenças entre grupos $[\mathrm{F}(3,44)=1,05, \mathrm{p}=0,38]$. Observou-se diferença significante entre blocos $[\mathrm{F}(6,18)=13,12, \mathrm{p}<0,001]$ e o teste Post Hoc de Tukey registrou as seguintes diferenças: o último bloco de tentativas da fase de aquisição foi mais preciso que os primeiros blocos dos testes $\mathrm{T} 1$ e T2, segundo bloco do teste $\mathrm{T} 2$ e que os dois blocos da retenção $(\mathrm{p}<0,05)$; o primeiro bloco do $\mathrm{T} 1$ foi menos preciso que o segundo bloco do $\mathrm{T} 1$, segundo bloco do $\mathrm{T} 2$ e que os dois blocos da fase de retenção ( $\mathrm{p}<0,001)$; o primeiro bloco do T2 foi menos preciso que o segundo bloco do $\mathrm{T} 2$ e que os dois blocos da retenção $(\mathrm{p}<0,001)$.

Foi também observada interação entre grupos e blo$\cos [\mathrm{F}(18,264)=1,68, \mathrm{p}<0,05]$ e o teste Post Hoc de Tukey registrou as seguintes diferenças: o grupo B-A foi mais preciso no último bloco de tentativas da fase de aquisição que em seu primeiro bloco do teste de retenção $(p<0,01)$; o grupo A-B foi mais preciso que o grupo B-A no primeiro bloco do teste de retenção $(p<0,01)$; o primeiro bloco do T1 do grupo $\mathrm{B}-\mathrm{B}$ foi mais preciso que o seu segundo bloco $(\mathrm{p}<0,05)$; o segundo bloco do T2 do grupo B-B foi mais preciso que o seu primeiro bloco $(p<0,01)$.

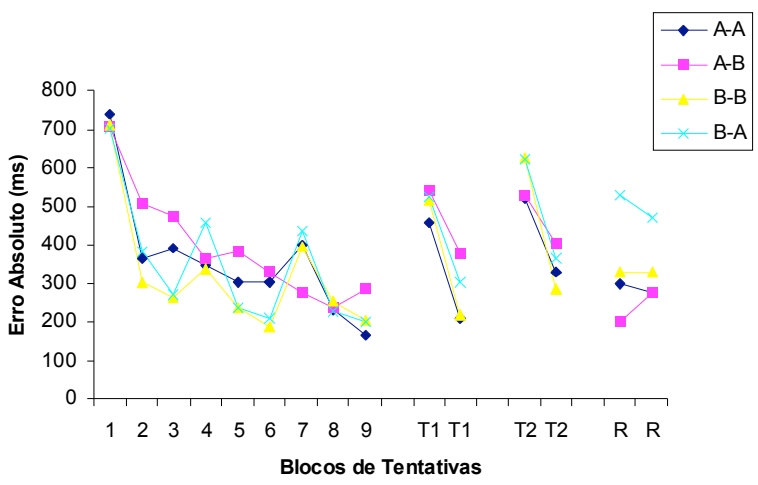

Figura 2. Erro absoluto dos grupos experimentais nas fases de aquisição e testes.

\section{Desvio-padrão do erro absoluto}

Para fase de aquisição (Figura 2), foi conduzida uma ANOVA two-way (4 grupos x 9 blocos) com medidas repetidas no segundo fator que indicou diferença significante entre grupos $[\mathrm{F}(3,44)=3,24, \mathrm{p}<0,05]$ e o teste Post Hoc de Tukey registrou maior variabilidade do grupo A-B sobre B-B $(p<0,05)$. Observou-se também diferença significante entre blocos $[\mathrm{F}(8,24)=4,25, \mathrm{p}<0,01]$ e o teste Post Hoc de Tukey indicou maior variabilidade, com exceção do $4^{\circ}$ bloco de tentativas, do primeiro para os demais blocos $(\mathrm{p}<0,01)$. Houve ainda interação entre grupos e blo$\cos [\mathrm{F}(24,352)=2,06, \mathrm{p}<0,01]$ e o teste de Post Hoc Tukey registrou as seguintes diferenças: maior variabilidade do grupo A-B sobre os grupos B-A $(p<0,001)$ e $B-B(p<0,05)$ no terceiro bloco de tentativas; maior variabilidade do primeiro sobre o último bloco do grupo A-A $(\mathrm{p}<0,01)$; maior variabilidade do primeiro sobre o $2^{\circ}, 3^{\circ}, 5^{\circ}, 6^{\circ}, 8^{\circ}$ e último bloco do grupo B-B $(p<0,01)$.

Para a comparação entre o último bloco de tentativas da fase de aquisição e os testes foi conduzida uma ANOVA two-way (4 grupos x 7 blocos) com medidas repetidas no segundo fator que não encontrou diferença significante para grupos $[\mathrm{F}(3,44)=1,57, \mathrm{p}=0,209]$. Entretanto, observou-se diferença para blocos $[\mathrm{F}(6,18)=9,08, \mathrm{p}<0,001]$ e o teste Post Hoc de Tukey registrou as seguintes diferenças:o último bloco de tentativas da fase de aquisição apresentou menor variabilidade que os primeiros blocos de tentativas dos testes T1 e T2 ( $<<0,01)$; o primeiro bloco do $\mathrm{T} 1$ apresentou maior variabilidade que o segundo bloco desse mesmo teste, primeiro e segundo blocos do $\mathrm{T} 2$ e que os dois blocos da retenção $(\mathrm{p}<0,01)$; o segundo bloco do T1 apresentou menor variabilidade que o primeiro bloco do T2 ( $<<0,01)$; o primeiro bloco do T2 foi mais variável que o segundo bloco desse mesmo teste e que os dois blocos da retenção $(p<0,01)$.

Houve também interação entre grupos e blocos $[\mathrm{F}(18,264)=2,12, \mathrm{p}<0,01]$ e o teste Post Hoc de Tukey indicou as seguintes diferenças: maior variabilidade do primeiro sobre o segundo bloco de tentativas no T1 do grupo A-A $(\mathrm{p}<0,001)$ e grupo B-B $(p<0,05)$; maior variabilidade do primeiro sobre o segundo bloco do T2 no grupo B-B $(\mathrm{p}<0,001)$. 


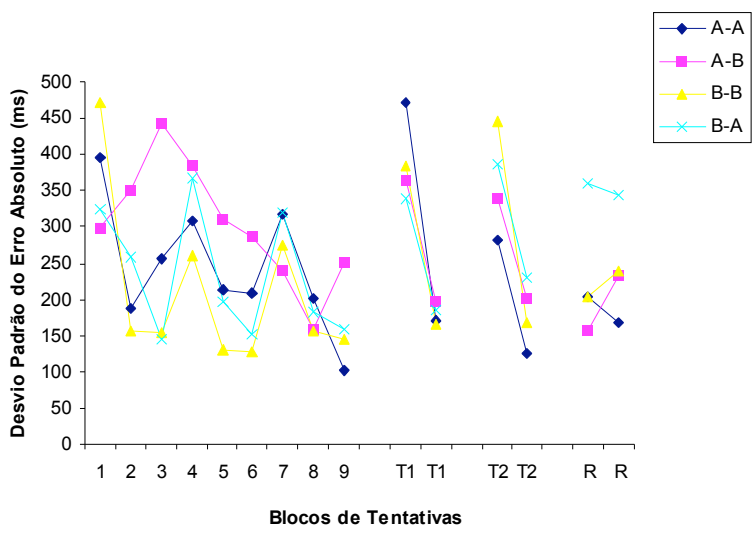

Figura 3. Desvio-padrão do erro absoluto nas fases de aquisição e testes.

\section{DISCUSSÃO E CONCLUSÃO}

O objetivo do estudo foi verificar o efeito da interferência contextual na aprendizagem de uma tarefa de posicionamento manual em idosos.

Os resultados mostraram que houve considerável queda no erro absoluto e aumento na consistência entre os grupos no decorrer da fase de aquisição, confirmando a melhora no desempenho já observada em outros estudos $(6,8,9)$.

Em relação à variabilidade, os grupos apresentaram consistência semelhante no último bloco de tentativas da fase de aquisição. Entretanto, na análise de toda a fase de aquisição, o grupo A-B apresentou-se mais variável que o grupo B-B. Assim como mostrou-se mais variável que os grupos que praticaram em blocos, B-B e B-A, no terceiro bloco de tentativas, resultado que está de acordo com Del Rey(6) e os pressupostos do EIC de forma geral.

Nos estudos que confirmam o EIC, o grupo de prática aleatória apresenta melhor desempenho nos testes, principalmente naqueles em que é exigida uma maior complexidade da tarefa $(3,4,6,8,18)$. No presente estudo os grupos apresentaram desempenho semelhante durante os testes de transferência.

Em relação à igualdade de desempenho verificada nos testes de transferência, vale ressaltar que houve uma tendência entre os sujeitos, quando foi retirado o feedback e apresentado novas tarefas, de focalizarem a atenção na correta execução da seqüência, sacrificando a precisão temporal em prol da precisão espacial. Segundo Larish e Stelmach( ${ }^{(9)}$ essa população tende a valorizar aspectos espaciais, como a cor- reta seqüência, em detrimento da precisão temporal ou velocidade de execução. Logo, essa estratégia pode ter contribuído para igualar os grupos durante os testes de transferência.

A interpretação dos resultados dos testes de transferência, quando utilizados nos estudos, ainda não está clara. Segundo Schmidt(13), os efeitos da manipulação da prática poderiam influenciar os aspectos da aprendizagem tanto na retenção quanto na transferência. Magill e Hall(10) confirmam que quando os efeitos do EIC são encontrados na retenção tendem a aparecer também nos testes de transferência. Por sua vez, Sekiya e Magill(15) confirmaram o EIC nos testes de retenção e transferência, somente quando houve manipulação de parâmetros. Eles afirmam que seus resultados são limitados ao delineamento utilizado, pois a transferência carece de mais estudos e pode depender do que está sendo variado na prática e mensurado no estudo, como parâmetros ou programas. O presente estudo variou programas durante a prática e segundo Magill e Hall(10), o EIC tende a ser confirmado quando se procede dessa maneira. Entretanto, uma série de trabalhos tem contestado essa afirmação, sugerindo que a variação de parâmetros seria a condição mais provável para verificar os efeitos da interferência contextual (15, 16, 17, 19).

Mesmo assim, os resultados mostraram uma dificuldade de adaptação do grupo que praticou em blocos durante a aquisição e foi exigido em um regime aleatório na fase de retenção, grupo B-A. Este grupo apresentou uma queda no desempenho durante $o$ primeiro bloco da retenção, quando comparado com seu último bloco de tentativas da fase de aquisição. Observou-se também superioridade do grupo A-B sobre o B-A no primeiro bloco do teste de retenção. Esses resultados estão de acordo com o trabalho de Shea e Morgan(18) e Carnahan, Vandervoort e Swanson ${ }^{(4)}$ e demonstram certa rigidez da estrutura de prática em blocos para se adaptar a uma nova condição de prática. No sentido contrário, observa-se uma maior capacidade do grupo de prática aleatória em se ajustar a uma nova condição de prática. Del Rey(6), ao contrário do presente estudo, verificou na fase de retenção uma menor precisão e consistência do grupo que praticou de forma aleatória durante a aquisição. Os resultados do teste de retenção contribuem para conclusão de um efeito parcial da interfe- 
rência contextual e vai ao encontro de outros resultados observados na literatura $(4,11,23)$.

Sob uma perspectiva de desenvolvimento, o EIC em idosos não tem sido consistentemente investigado. De forma geral, os poucos estudos realizados comprovaram parcialmente $(4,6,8)$ os efeitos benéficos da prática aleatória para essa população. A análise dos resultados dos estudos encontrados na literatura e os do presente estudo não apresenta evidências claras do EIC em idosos em todos os contextos de testes. Pode-se destacar os efeitos positivos da prática aleatória em idosos em alguns aspectos dos testes de retenção. Dele Rey(6) e Carnahan e colegas(4) encontraram um maior efeito de aprendizagem por parte dos sujeitos do grupo de prática aleatória somente nas tarefas que esses autores definiram como as mais difíceis. Esses aspectos foram a velocidade do estímulo(6) e a ordem espacial dos eventos(4). No presente estudo, um fator que confirma parcialmente os efeitos da interferência contextual em idosos em aspectos de maior exigência, foi a melhor adaptação do grupo A-B comparado ao B-A no $1^{\circ}$ bloco da retenção quando os mesmos praticaram as mesmas habilidades exigidas na aquisição, porém em uma outra estrutura de prática.

Resultados inconsistentes sobre o EIC vêm também sendo encontrados quando os sujeitos analisados se encontram em outros níveis de desenvolvimento. De acordo com Ugrinowitsch e Manoel(22), estudos realizados com crianças tendem a não confirmar o EIC, enquanto um maior número de confirmações é encontrado em pesquisas com sujeitos adultos.

Esses resultados sugerem que muito por ser esclarecido em relação ao EIC e níveis de desenvolvimento. Em suma, o presente estudo fornece subsídios para se confirmar parcialmente o efeito da interferência contextual em idosos, mostrando maior potencial de adaptação a um novo regime de prática do grupo que praticou de forma aleatória. Seria interessante a investigação de outras questões envolvendo o EIC e idosos, como distinção na aprendizagem de parâmetros e programas e complexidade da tarefa. Assim sendo, novos estudos que investiguem o EIC com idosos precisam ser realizados.
CORRESPONDÊNCIA Wesley Rodrigo Gonçalves

Rua Monsenhor João Martins, 2144

Bairro Novo Progresso - Contagem / MG

32140-470 Brasil

e-mail: wesleyrgoncalves@hotmail.com 


\section{REFERÊNCIAS}

1. Arnone-Bates M, Hebert E, Titzer R (1999). The contextual interference effect with children learning an applied task. Research Quarterly for Exercises and Sport 70: a-65.

2. Battig WE (1979). The flexibility of human memory. In: LS Cermak, FI Craik (eds.). Levels of processing in human memory. Hillsdale, NJ: Lawrence Erlbaum, 23-44.

3. Bortoli L, Robazza C, Durigon V, Carra C (1992). Effects of contextual interference on learning technical sports skills. Perceptual and Motor Skills 75: 555-562.

4. Carnahan H, Vandervoort AA, Swanson LR (1993). The influence of aging on motor learning. In: G Stelmach (ed.). Sensoriomotor impairments in the elderly. Dordrecht: Kluver, 41-56.

5. Corrêa UC (1997). Interferência Contextual: Contribuições à aprendizagem motora. In: AM Pellegrini (ed.). Coletânea de Estudos: Comportamento motor. São Paulo: Movimento, 129-158.

6. Del Rey P (1982). Effects of contextual interference on the memory of older females differing in levels of physical activity. Perceptual and Motor Skills 55:171-180.

7. Dick MB, Beth RE, Shankle RW, Dick-Muehlke C, Cotman CW, Kean ML (1996). Acquisition and long-term retention of a gross motor skill in Alzheimer disease patients under constant and varied practice conditions. Journal of Gerontology 51:103-111.

8. Dick MB, Hsieh S, Dick-Muehlke C, Davis DS, Cotman CW (2000). The variability of practice hypothesis in motor learning: does it apply to Alzheimer's disease? Brain and Cognition 44: 470-489.

9. Larish DD, Stelmach GE (1982). Preprogramming, programming, and reprogramming of aimed hand movements as a function of age. Journal of Motor Behavior 14: 322-340.

10. Magill RA, Hall KG (1990). A review of the contextual interference effect in motor skill acquisition. Human Movement Science 9: 241-289.

11. Pollatou E, Kioumourtzoglou E, Angelousis N, Mavromatis G (1997). Contextual interference effects in learning novel motor skills. Perceptual and Motor Skills 84: 487-496.

12. Santos S, Tani G (1995). Tempo de reação e a aprendizagem de uma tarefa de "timing" antecipatório em idosos. Revista Paulista de Educação Física 9: 51-62.
13. Schmidt RA (1975). A schema theory of discrete motor skill learning. Psychological Review 82: 225-260.

14. Schmidt RA, Wrisberg CA (2001). Aprendizagem e performance motora: uma abordagem da aprendizagem baseada no problema ( $2^{\mathrm{a}}$ ed.). Porto Alegre: Artmed.

15. Sekiya H, Magill RA (2000). The contextual interference effect in learning force and timing parameters of the same generalized motor program. Journal of Human Movement Studies 39: 45-71.

16. Sekiya H, Magill RA, Anderson DI (1996). The contextual interference effect in parameter modifications of the same generalized motor program. Research Quarterly for Exercises and Sport 67: 59-68.

17. Sekiya H, Magill RA, Sidaway B, Anderson DI (1994). The contextual interference effect for skill variations from the same and different generalized motor programs. Research Quarterly for Exercises and Sport 65: 330-338.

18. Shea JB, Morgan RL (1979). Contextual interference effects on the acquisition, retention, and transfer of a motor skill. Journal of Experimental Psychology: Human Learning and Memory 5: 179-87.

19. Silva AB, Lage GM, Gonçalves WR, Palhares LR, Ugrinowitsch H, Benda RN (2004). Contextual interference and manipulation of generalized motor program and parameters in timing tasks. Journal of Sport $\mathcal{E}$ Exercise Psyhology 26: S173.

20. Spirduso WW (1980) Physical fitness, aging, and psychomotor speed: a review. Journal of Gerontology 35: 850-865.

21. Spirduso WW (1975). Reaction and movement time as a function of age and physical level. Journal of Gerontology 30: 435-440.

22. Ugrinowitsch H, Manoel EJ (1999). Interferência contextual: Variação de programa e parâmetro na aquisição da habilidade motora saque do voleibol. Revista Paulista de Educação Física 13: 197-216.

23. Wegman E (1999). Contextual interference effects on the acquisition and retention of fundamental motor skills. Perceptual and Motor Skills 88: 182-187.

24. Wrisberg CA, Liu Z (1991). The effect of contextual variety on the practice, retention, and transfer of an applied motor skill. Research Quarterly for Exercises and Sport 62: 406-412. 